\title{
Quimioterapia Neoadjuvante para o Tratamento do Câncer de Mama. Avaliação Clinica, Imunológica e Psicológica Comparada ao Índice de Resposta
}

Autor: Carlos Francisco Erbolato Melo

Orientador: Prof. Dr. Carlos Roberto de Resende Miranda

Dissertação apresentada à Faculdade de Ciências da Saúde da Universidade de Brasília, para obtenção do Título de Mestre em Ciências da Saúde em 8 de agosto de 1999.

Para avaliar a imunidade celular e a depressão, estudamos 21 pacientes com câncer de mama, nos estadios IIb e III submetidas a avaliação antes e depois de tratamento quimioterápico neoadjuvante. Utilizamos 3 ciclos de 5 -FU $500 \mathrm{mg} / \mathrm{m}^{2}$, adriamicina $50 \mathrm{mg} / \mathrm{m}^{2}$ e ciclofosfamida $500 \mathrm{mg} / \mathrm{m}^{2}$. A avaliação imunológica foi realizada através do Teste Cutâneo de Hipersensibilidade Retardada (TCHR). A avaliação da depressão se deu através do Inventário Beck de Depressão (BDI). Encontramos niveis de intensidade ao TCHR significativamente menores nas pacientes com câncer de mama, quando comparadas a controles sem câncer $(\mathrm{p}<$ 0,05). Não houve correlação entre o TCHR e o Índice de Resposta Objetivo (IRO). Os resultados do TCHR não se alteraram significativamente com a quimioterapia. A presença de depressão indicada pelo BDI ocorreu em $30 \%$ das pacientes, antes da quimioterapia, e em $45 \%$ das pacientes, após o término do tratamento. Não houve correlação entre os niveis de depressão e o IRO. Dentre as pacientes previamente deprimidas, houve melhora significativa dos niveis de depressão total e do indicador cognitivo-afetivo $(p<0,05)$, após o tratamento. A sensação de estar recebendo tratamento parece ser o motivo para melhora dos indicadores psicométricos nas pacientes deprimidas. O TCHR e os niveis gerais de depressão obtidos através do BDI não foram capazes de predizer a resposta ao tratamento quimioterápico neoadjuvante. A busca de fatores preditivos ao tratamento do câncer de mama é dificultada pela complexidade da fisiopatologia deste tipo de tumor, e pelas dúvidas etiológicas que o cercam.

Palavras-chave: Mama: câncer. Depressão. Quimioterapia.

\section{Monitorização Ambulatorial da Pressão Arterial em Gestantes Normotensas: Estudo Longitudinal do Ritmo Pressórico e da Frequência Cardiaca}

Autor: Antônio Vieira Machado

Orientador: Prof. Dr. Victor Hugo de Melo

Tese apresentada à Faculdade de Medicina - Universidade Federal de Minas Gerais, para obtenção do título de Doutor em Ginecologia, em 30 de abril de 1999.

O conhecimento e, principalmente, o entendimento da pressão arterial (PA) constituem uma busca constante dos pesquisadores e estudiosos, pois a sua mensuração é de grande importância na assistência à gestante durante o pré-natal. A Monitorização Ambulatorial da Pressão Arterial (MAPA) tem-se revelado uma técnica acurada e reprodutivel na mensuração da PA por 24 horas, de forma contínua, automática e sem interrupção das atividades diárias do indivíduo, bem como durante o sono. Este estudo visa mostrar, longitudinalmente, os perfis e os parâmetros do ritmo pressórico e da freqüência cardíaca de gestantes normotensas nos três trimestres da gestação. O estudo, longitudinal e aleatório, contemplou 23 gestantes normotensas, com idade média de 23,3 $\pm 3,9$ anos e com uma MAPA em cada trimestre (até 14 semanas, entre 18 e 28 semanas e entre 32 e 40 semanas, pri- meiro, segundo e terceiro trimestre, respectivamente), utilizando o monitor SpaceLabs, modelo 90207. Observou-se um aumento significativo $(\mathrm{p}<0,01)$ nas pressões arteriais sistólica, diastólica e média de 24 horas, diurna e noturna no terceiro trimestre da gestação. A pressão arterial e a freqüência cardíaca materna diurnas foram significativamente maiores que as noturnas, nos três trimestres. A freqüência cardiaca materna não se alterou com o evoluir da gestação. Demonstramos a presença do ritmo circadiano da pressão arterial e da freqüência cardíaca materna durante a gestação normal, bem como aumento da pressão arterial no terceiro trimestre da gestação.

Palavras-chave: Gravidez normal. Monitorização ambulatorial da pressão arterial. Ritmo circadiano. 